\title{
Comparison of Burden on Youth in Communicating with Elderly using Images Versus Photographs
}

\author{
Miyuki Iwamoto \\ Graduate School of Engineering and \\ Science, Kyoto Institute of \\ Technology \\ Kyoto, Japan
}

\author{
Noriaki Kuwahara \\ Graduate School of Engineering and \\ Science, Kyoto Institute of \\ Technology \\ Kyoto, Japan
}

\author{
Kazunari Morimoto \\ Graduate School of Engineering and \\ Science, Kyoto Institute of \\ Technology \\ Kyoto, Japan
}

\begin{abstract}
Conversation is a good preventative against behavioral problems in the elderly. However, caregivers are usually very busy tending to patients and lack the time to communicate extensively with them. Toward overcoming such problems actively listening volunteers have more opportunities to communicate with the elderly, but the number of skilled volunteers is limited. Therefore, we investigated conversational support systems for inexperienced volunteers; such systems usually include content such as photographs, videos, and music. We expected that the volunteers would feel less stress when using videos instead of photographs for conversational support because the former provided both volunteers and patients with richer information than the latter. On the other hand, photographs gave patients more chances to talk with volunteers. However, there has been no research to date on the effect of content type upon stress and conversational quality. In this paper, we compared using photographs with using video from such viewpoints.
\end{abstract}

Keywords-elderly; reminiscence videos; senior care home photographic image

\section{BACKGROUND AND PURPOSE}

Japanese society is recently facing the problem of having a "super-aging" population. The proportion of aged people is growing. The population over 65 years old, so-called "BabyBoomers," is now over 30 million, and it is anticipated to be 36 million in 2030. [1]

The rate of families consisting of old couples and old singles is increasing. In some cases s/he may pass an entire day without speaking a word, which can lead to a disuse of cognitive functions and a heightened risk of dementia and/or depression.

We need to understand such physical and psychological characteristics in our communication with aged people. For example, we must respect the damage to their pride at losing their visual, auditory, and cognitive functions. Experts such as clinical psychologists, therapists, or listening volunteers may be able to deal with these issues in their communication efforts. However, their numbers are insufficient to meet the current needs.

Thus, the younger generations are expected to be talking partners for aged people, but there is a problem in that they are unfamiliar with how to communicate with the elderly because the vast majority/ many grew up in small families without grandfathers or grandmothers.
There have been some attempts to solve the problem using a picture or a video as a trigger for conversation. But there is no research regarding the mental burden felt by the partner students/ volunteers. That means the research has concentrated on communication support systems to improve QOL for aged people, while the mental burden of the young partners has been neglected. Some volunteers have said that in their experiences they felt less mental burden in watching a video than in looking at a photo. Yet the effects in quality and in quantity of conversation based on the contents of the support system have not been examined. It is therefore unclear whether there is a difference between how photos and videos affect their mental burden.

We have also used photographs and videos as supporting tools for communication between aged people with dementia and students/ volunteers, as shown in the following figure. We have noticed that although these materials make communication smoother and offer a valuable opportunity for the aged people, some degree of mental burden is imposed upon the students/ volunteers.

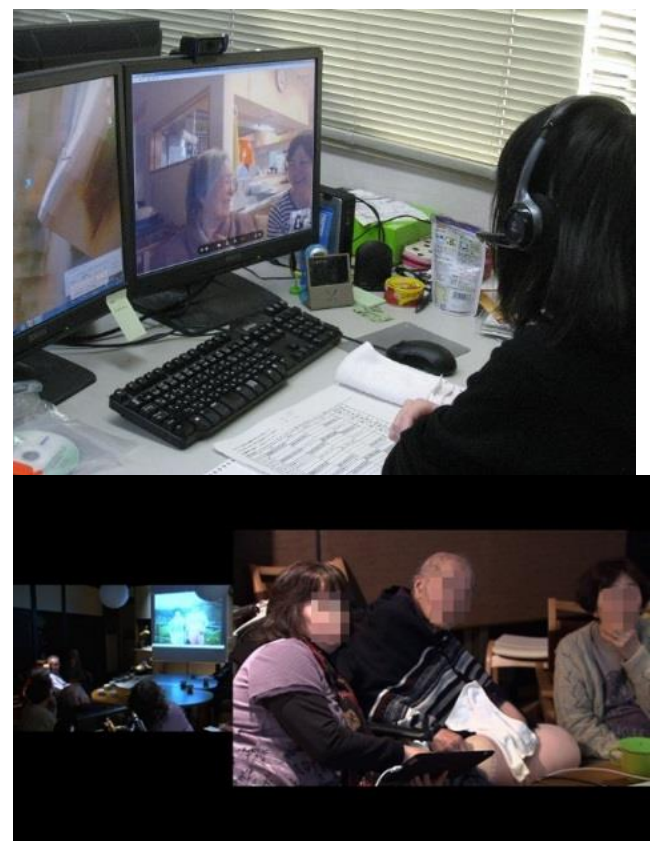




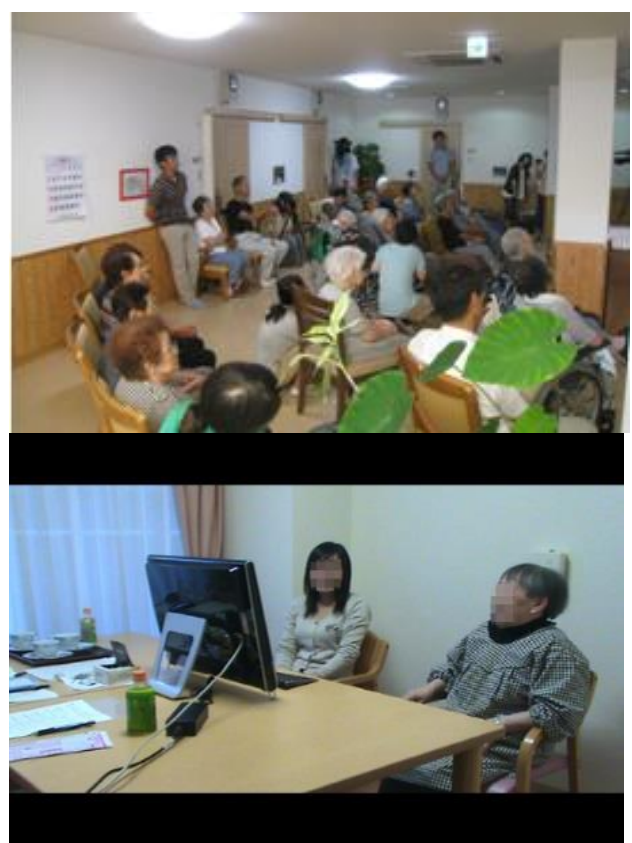

Fig. 1. Volunteers utilizing conversation support content

Many caregivers and volunteers untrained in listening often feel considerable mental burden. There is some research on promoting conversation between patients and caregivers or volunteers, or among patients, by providing topics and/ or sharing photos or videos. (Exam. [2][3][4]) This focused on what is known as reminiscence technique, which is effective for controlling dementia and reducing the mental burden placed upon the partner students/ volunteers. We call the information media which provide the topics (hereafter, "media") "communication support content."

The media in general include photos, videos, and music. Their effectiveness with regard to the mental burden felt by caregivers, or the quality of communication between patients and caregivers, is unknown.

We examined the differences in the mental burden and the quality of communication between patients and caregivers/ volunteers when they used photos versus when they used videos as communication support content in order to find the best medium for communication.

We measured the mental burden in reference to our research presented at the Human Interface symposium [6]. We compared whether less burden was felt in communication using photos or videos, improving upon problems found in the former research. As for quality and quantity of communication, we estimated these by the length and sight lines observed in the communicative exchange, an approach used in the 5-stage subjective assessment from previous conversational support studies. [2]

We found in the previous research that measuring the mental burden was difficult because of the individual characters of the aged people. In that project, a pair of students would face a patient with each having the chance to talk only once. We changed the situation in this case such that each student interviewed five patients. We could then evaluate the mental burden by averaging the influence of compatibility between the patient and the partner.

There was an additional problem concerning the accuracy of the length of conversation, as it could be interrupted by searching for a photo or video via the internet in the previous research. This was solved by preparing the photos and videos in advance.

The subjectivity of our questions and video recording was furthermore an issue. . Therefore we added measuring heart rates as an objective aspect this time.

Finally, the variation in content when students searched for photos or videos on the internet was also an issue. This could present difficulty in measuring the degree of mental burden because the photo or video may or may not have been interesting or favorable to them. In the case of the present research, we used the same content in order to control the results measuring the quality of the conversation.

We conducted an experiment to improve upon these points.

\section{EXPERIMENT}

\section{A. Outline}

We eliminated art-related topics because there was a great generation gap between the patients and students, and some topics were thought improper. We chose the topic categories of food, events, and plays from the former experiment. As for subjects, one student interviewed five patients and we estimated an average of the mental burden while excluding personal compatibilities.

We measured the length of conversation, preparing the photos and videos in advance to eliminate the burden of having to search for these during the conversation. We counted the number of heartbeats as an objective marker adding to the subjective estimation of mental burden.

The content of photos and videos in the categories of food, events, and plays were provided in advance. The photos were still pictures from the videos to examine the quality and quantity of the conversation and the related mental burden. Using the same content allowed us to control the influence of difference in contents.

The questions from the 5-stage subjective assessment were applied to estimate the quality of conversation after each experiment, as we had done before. We measured the quantity by the length of the communicative exchange in the video recording and looking at sight lines.

\section{B. Evaluation items}

We carried out checks on the degree of burden resulting from the conversation upon the students. A stress check board was positioned out of sight of the elderly patients (Fig.2). The numbers "1-7" were written on this stress check board, with " 1 " equating to no (stress) burden during the conversation, and "7" meaning that a great deal of stress was felt. At the beginning of the experiment, the students' stress levels were at "1". The students' stress levels were then measured each minute, at which time students could chose " $1-7$ " according to 
the degree of stress they were experiencing during the conversation.

This represents the degree of stress felt in the conversation at a given time. During the conversation, the facial expressions and movements (appearance and nods) of the students and the elderly were recorded. We also measured the students' heart rates in order to determine their conversational stress levels.

During the evaluation of the conversational quality, a questionnaire was given once during each experiment according to the fifth stage subjective evaluation.

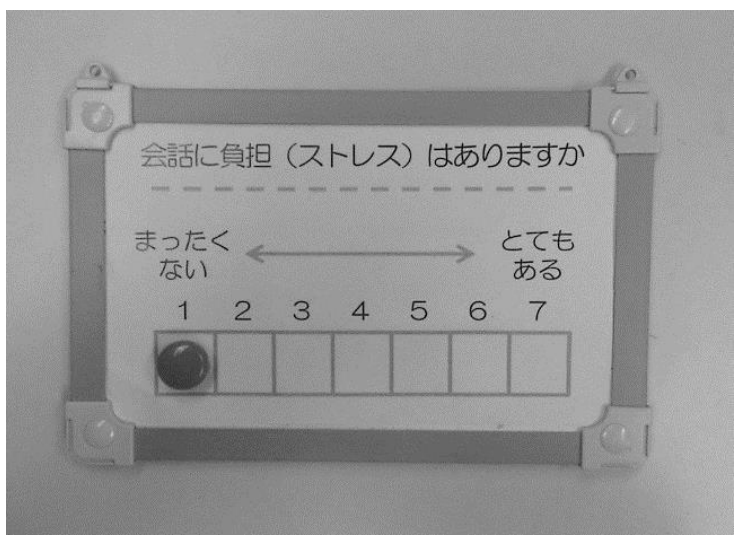

Fig. 2. Stress Check Board

\section{Experiment environment}

The layout of the experiment environment is shown in Figures 3 and 4 below. We borrowed a nursing home room, in which we placed chairs side by side. We used a desktop PC to which we uploaded photos and videos fitting into the abovementioned categories in order to facilitate the 10 minuteconversation. We asked the student to point to the appropriate number on the check board in a way hidden from the patient's eyes, preventing the student from drawing the patient's attention while they were sitting side by side.

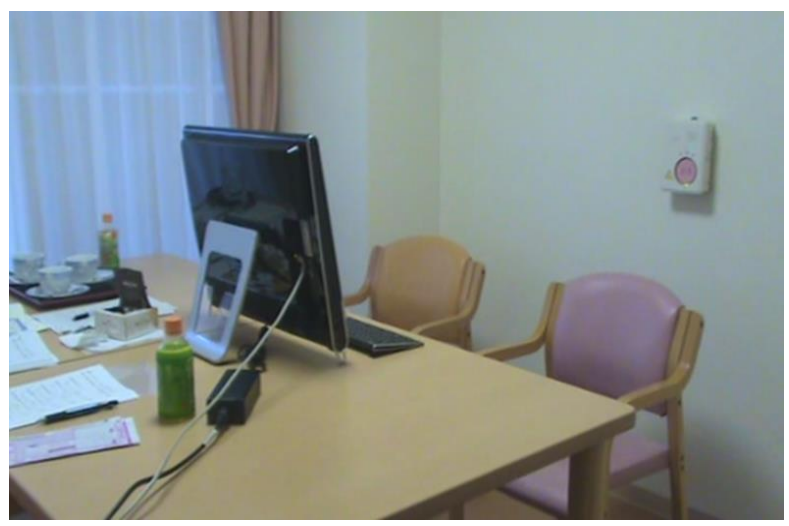

Fig. 3. Experiment environment

\section{The Subject}

The participants were 2 students, both of whom were 23 years old. Their degree of interpersonal skill was diagnosed in advance by the Yatabe-Guilford sociability personality diagnostic test. The patients were 8 senior ladies, ages 82 to 90 , who were suffering from mild dementia.

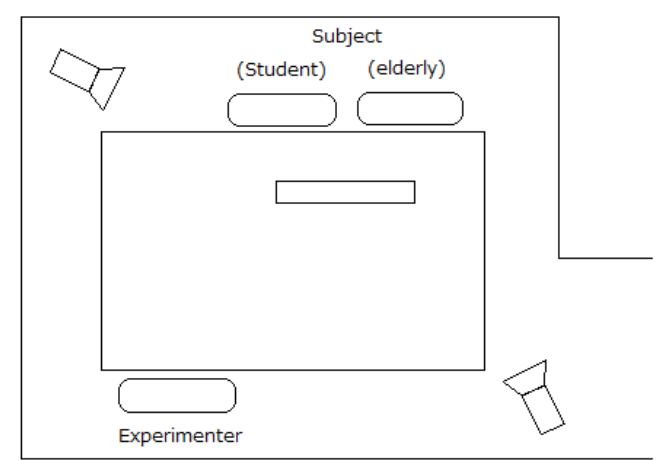

Fig. 4. The layout of the experimental environment

\section{E. Procedure}

The students and the patients talked while viewing a photo or a video through the PC. We prepared 20 photos each for the categories food, events, and plays, and these were shown through the flow of the conversation. We prepared a 10-minute video which was displayed on the PC monitor throughout the 10-minute conversation.

The student indicated the degree of mental burden on the stress check board each minute. The board was hidden from the patient's eye. Our purpose was to check the mental burden felt by the student during the conversation, not to check their complaints regarding the patient. Thus, the student was able to point out their feeling without having to be concerned about the patient. They could declare their feeling honestly. The board was sometimes shown in the middle of their conversation but they continued to talk at the moment they pointed. They answered the questions from the 5-stage subjective evaluation each time after the experiment. The heart rates of the participant students were also checked in each case to measure their mental burden. They placed the counters on their bodies in advance.

\section{RESULT}

Fig.5 shows the stress level of Student A and B (simply A or B below). The horizontal axis represents the photos and videos of subjects $A$ and $B$, respectively, and the vertical axis represents the accumulation on the numerical stress check board. This is a comparison of the students' stress levels in the case of looking at photos and in the case of watching videos when they felt a sense of mental burden upon continuing the conversation using support contents.

From the diagnosis of characters, A showed $58 \%$ interpersonal skill and $50 \%$ sociability while B showed $75 \%$ and $61 \%$, respectively. The students showed a difference in interpersonal skill. We compared the results of their mental burden from the conversations. A seemed to feel more mental burden when he showed photos to the patient.

We counted the heart rates of $\mathrm{A}$ and $\mathrm{B}$ and we took averages of the former half and the latter half in the 10-minute conversation with each subject. The vertical axis in Fig.6 and in Fig.7 show the number of heartbeats counted [number/ minute], and the horizontal axis show the experiment times in the former half and the latter half. 


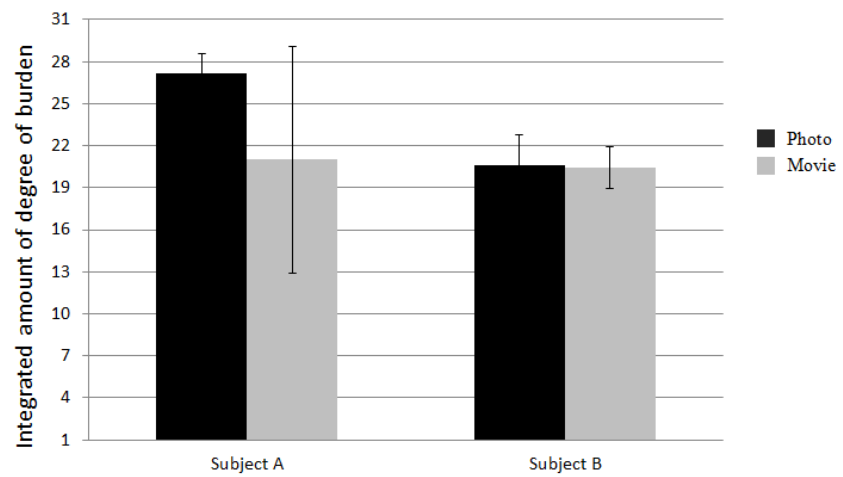

Fig. 5. Integration of the 10 minutes of degree of burden

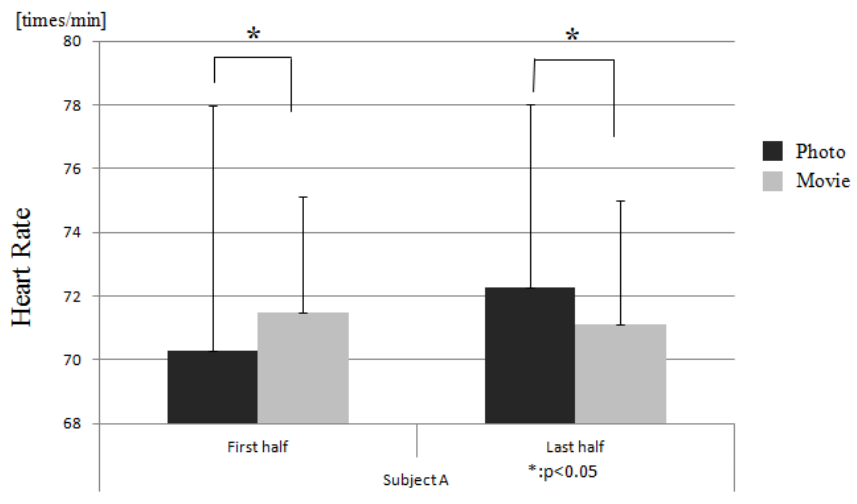

Fig. 6. The first half for Subject A, the average heart rate for the second half

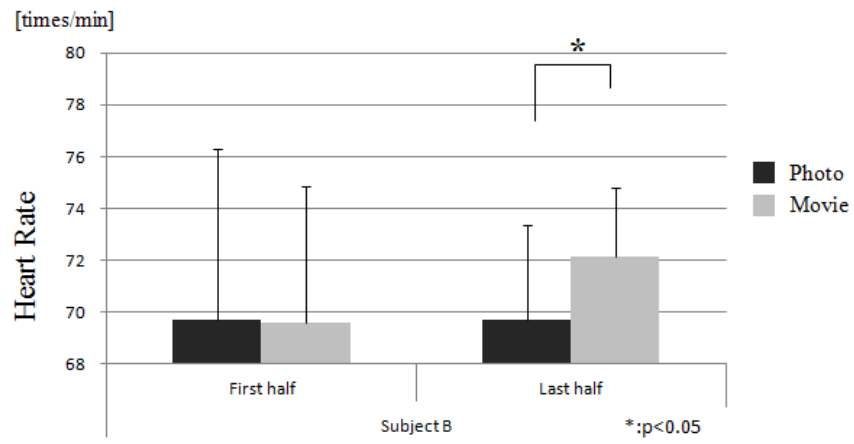

Fig. 7. The first half for Subject B, the average heart rate for the second half

We found no substantial change in the heart rates of A either in the former half or the latter half, as shown in Fig.6. In the former half, his heart rate was higher when showing videos, but in the latter half, it was higher when showing photos. In neither the former nor latter half was a significant difference in hear rate observed in the cases of photos or videos as a result of the (significance level 5\%) t-test.

The heart rate of $\mathrm{B}$ had no difference at the beginning either in showing photos or in showing videos. In the latter half, his heart rate showed a greater change when showing photos than when showing videos. There was a significant difference by $\mathrm{t}-$ test in his heart rate between showing photos and showing videos.

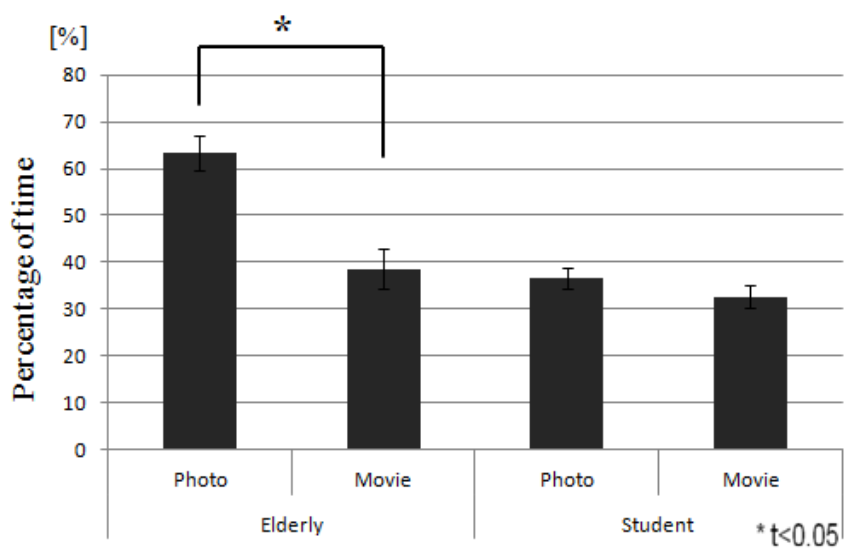

Fig. 8. Speech Time (Subject A)

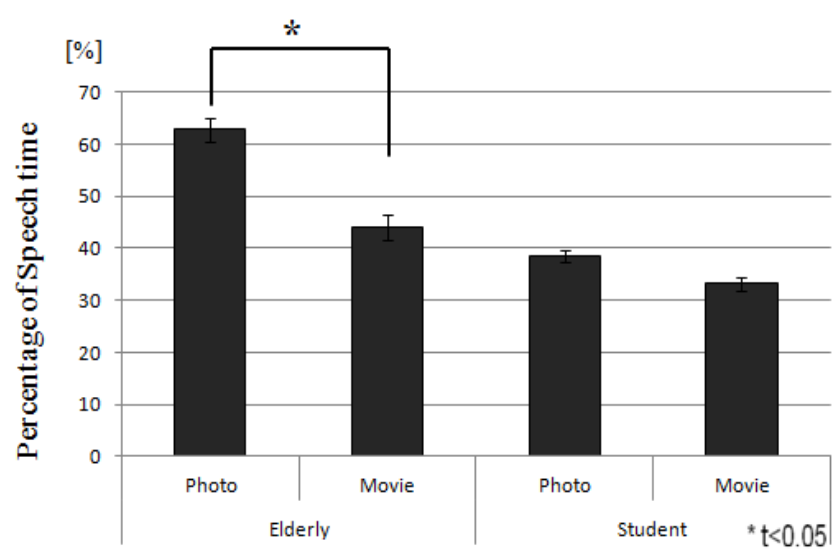

Fig. 9. Speech Time (Subject B)

Fig.8 (A) and Fig.9 (B) are the results of conversation time counted from the video recording when showing photos and when showing videos. The vertical axis is the rate (\%) of the length of the communicative exchange over the 10 minutes, and the photos and the movies of the student and those of the patient. These figures show that either $\mathrm{A}$ or $\mathrm{B}$, or the patient or the partner, talks longer when showing photos than when showing videos, though the difference in the time of the communicative exchange on the part of the patient was larger than that of the student. The results of the t-test $(5 \%$ significance) were significantly different with regard to the length of the communicative exchange on the part of the patient and the student.

We also examined their sight lines during the conversation. "Sight line" here means the amount of time when they are looking at their interlocutor. In Fig.10, the vertical axis shows the proportion of time in the whole conversation for the subjects and the horizontal axis shows when the subject was looking at his interlocutor or the screen, for the patient and the student. In the case of showing photos, both the patient and the partner tended to look at their interlocutor. But in the case of showing videos, both of them tended to look at the screen. 


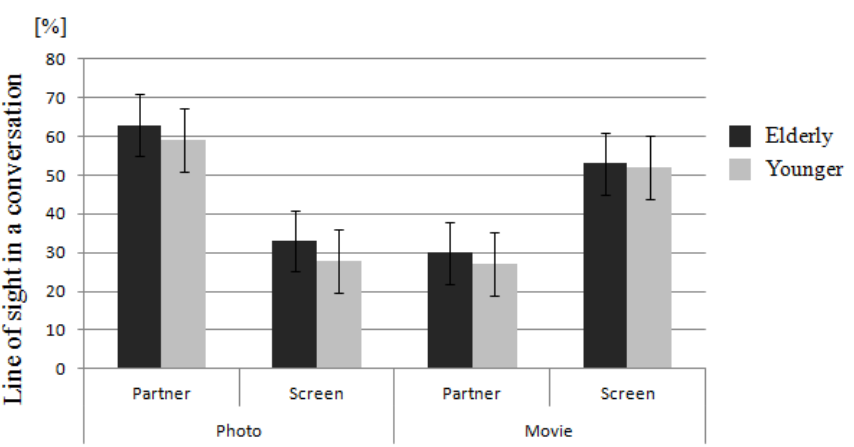

Fig. 10. Comparison of the line-of-sight during the speech time for the elderly and the young people

\section{CONSIDERATION}

We found that there was a difference in the length of the communicative exchange for patients when viewing photos versus viewing videos. This must be caused by the fact that patients tend to watch videos more than their listening partners, which made interfered in the conversation. We expected in such a case, the communicative exchange on the part of the partner would become more frequent and longer, but it did not. They showed almost the same time in their communicative exchange in photo viewing and in video viewing. Still, we found showing photos made both of them talk longer.

We consider that one of the reasons why the patients talked longer when viewing photos is, through our watching the recorded video of the experiment, the patients gazed at the photo and talked about the photo shortly, and after that they talked for a long time about unrelated things. On the contrary, the patients watched the videos for a longer time, and talked less. But the student partner felt more interested in the video, thinking that they had succeeded in providing the topic which made them feel less burdened.

Thus we conclude that showing photos made both the patients and the partners talk at greater length. We suggest that the patients could talk more deeply on the topic by showing photos considering the former results of subjective evaluations, and that the student partner, as in the former results of the subjective evaluation, feel less burdened by showing videos, leading them to talk more intimately.

\section{Challenges For the Future}

We must examine in the future the mental burden of the partner in a given communicative exchange or in searching for topics. We anticipate constructing a support system which changes the content from photo to video properly adapted to the situation to reduce the volunteer's mental burden.

As pointed out by the former study [4], when the students searched for photos or videos during the conversation, they felt that it was difficult to call a proper trigger word to mind. We suggest making the lists of each category like food, events, or play in order to remove the nuisance of searching for targets from an infinite set of data in YouTube or other pictures. Or at the point the list of the searched result is made, an easy previewing would help in choosing the one.

We intend to develop a touch panel system which would make searching for photos and/ or videos much easier.

\section{ACKNOWLEDGMENT}

This work was supported by JSPS KAKENHI Grant Number $15 \mathrm{H} 01698$

\section{REFERENCES}

[1] A Overview Ministry of Health, Labour and Welfare, a 25-year Heisei version of Annual Report on Health and Welfare,Japan's population trends, the Ministry of Health, Labour and Welfare website Appendices (online) < http://www.mhlw.go.jp/wp/hakusyo/kousei/13-2/dl/01.pdf> pp. 5

[2] Arlene J. Astell, Maggie P. Ellis, Lauren Bernardi, Norman Alm, Richard Dye, Gary Gowans, Jim Campbell, Using a touch screen computer to support relationships between people with dementia and caregivers;Interacting with Computers 22, pp.267-275(2010)

[3] Kuwahara Noriaki, Kuwahara Kazuhiro, Abe Shinji, Susami Kenji, Yasuda Kiyoshi; Video memories that utilize annotation of photo-Application and evaluation to persons with dementia - - making support; Artificial Intelligence Journal, Vol.20, No.6, pp.396-405 (2005)

[4] Tuji Airi, Kuwahara Noriaki, Morimoto Kazunari; Implementation of interactive reminiscence photo sharing system for elderly people by using web services; Human Interface Society, (2010)

[5] Takai Shota; Topic suggestions propulsion system of conversation during the first meeting in accordance with the TPO; Heisei 21 year master's thesis, (2010)

[6] Iwamoto Miyuki, Kuwahara Noriaki, Morimoto Kazunari; Comparison between the Burden of the Conversation by Using Photographic Image and that by Using Motion Video; Human Interface Society 2012, pp.579-584 (2012) 